\title{
The relationship between area deprivation and prescription of long-acting reversible contraception in women of reproductive age in Lothian, Scotland, UK
}

\author{
Catherine Rachel Morgan, ${ }^{1}$ Hanhua Liu ${ }^{2}$
}

\begin{abstract}
${ }^{1}$ General Practice, Tyne Medical Practice, Haddington, East Lothian, UK

${ }^{2}$ Research Fellow, Department for Occupational and Environmental Health, University of Manchester, Manchester, UK
\end{abstract}

\section{Correspondence to} Dr Catherine Rachel Morgan, Research Fellow, Tyne Medical Practice, Newton Port Surgery, Newton Port, Haddington, EH41 3NF,UK; catherine.morgan@ nhslothian.scot.nhs.uk

Received 8 June 2016 Revised 3 February 2017 Accepted 20 March 2017 Published Online First 4 May 2017
CrossMark

To cite: Morgan CR, Liu H. J Fam Plann Reprod Health Care 2017:43:281-288.

\begin{abstract}
Background Reducing unplanned pregnancy in Scotland is a key government objective. Long-acting reversible contraception (LARC) is a cost-effective way to reduce unintended pregnancy. Abortion and teenage pregnancy rates are highest in the most deprived areas. One possible explanation could be contraceptive prescribing inequality. This study examined the relationship between area deprivation measured by the Scottish Index of Multiple Deprivation and LARC prescription.

Methods Using Scottish electronic prescribing data from primary care and sexual and reproductive health clinics, this study analysed female Lothian residents with a valid postcode aged 16-49 years who received a contraceptive prescription from 1 April 2012 to 31 March 2014. Prescription of LARC (intrauterine, implant or injectable contraceptive) compared with nonLARC (oral pill, patch, ring or diaphragm) was examined. Logistic regression was performed adjusting for age group and prescription location.

Results A total of 90150 women were included; $21.1 \%$ of prescriptions were LARC and $15.3 \%$ VLARC (intrauterine method or implant). Women residing in the most deprived quintile (Q1) and prescribed contraception received a significantly higher proportion of LARC than quintiles 2-5 (Q2-5). Odds ratios compared with Q1 were: Q2 0.86, Q3 0.77, Q4 0.59 and Q5 0.51. Women in quintile 1 were also significantly more likely to receive $\mathrm{VLARC}$ than quintiles 2-5.
\end{abstract}

Conclusion Women in the most deprived quintile in Lothian who are prescribed contraception are significantly more likely to receive LARC and VLARC compared with women in less deprived quintiles.

\section{Key message points}

- Women living in the most deprived quintile in Lothian, Scotland who are prescribed contraception are significantly more likely to receive longacting reversible contraception (LARC) and very long-acting contraception ( $v L A R C)$ compared with women in less deprived quintiles.

- Achieving a higher proportion of LARC in women prescribed contraception in deprived areas does not necessarily result in reduced unintended pregnancies.

- Wider determinants of social inequalities such as education and employment also need to be targeted by policy to realise a narrowing of the inequality gap.

\section{INTRODUCTION}

Unintended pregnancy can have negative consequences for women, ${ }^{1}$ children $^{2}$ and society. ${ }^{3}{ }^{4}$ Prevalence is difficult to measure; current UK estimates suggest $16 \%$ of pregnancies are unplanned and 29\% 'ambivalent'. ${ }^{1}$ The majority occur in the age group 20-34 years and nearly half of teenage pregnancies are unplanned. ${ }^{1}$

Reducing unplanned pregnancy is a key objective of the Scottish Government. ${ }^{5}$ Scottish teenage pregnancy rates are declining but still higher than most Western European countries and strongly correlated with deprivation. ${ }^{6}$ Rates are 4.8 times higher in the most deprived compared with least deprived areas. ${ }^{6}$ In England the difference is six-fold. ${ }^{7}$ Pregnant teenagers in the most deprived areas 
have 12 times the delivery rate compared with the least deprived areas. ${ }^{6}$ Teenage births can perpetuate the cycle of socioeconomic deprivation, social exclusion and health inequality. ${ }^{7}$

Abortion rate can be an indicator of unplanned pregnancy. Scottish rates have fallen since $2008 .^{8}$ The highest rates are in age group 20-24 then 25-29 years. ${ }^{8}$ Areas of highest deprivation have $73 \%$ greater abortion rates than the least deprived areas; in Lothian, the rate is $90 \%$ higher. ${ }^{8}$ Over half of all abortions were for women residing in the two most deprived quintiles. ${ }^{8}$

Contraceptive use is high in the UK but the most popular methods are oral contraceptives [combined oral contraceptive pill (COCP), progestogen-only pill (POP)] and male condoms, both user-dependent methods. ${ }^{9}$ Long-acting reversible contraception (LARC) includes intrauterine methods [intrauterine device (IUD)/intrauterine system (IUS)], progestogen subdermal implants and progestogen injectable contraceptives, ${ }^{10}$ while non-LARC methods include oral contraceptives, condoms, patch, ring and diaphragm. The National Institute for Health and Care Excellence (NICE) concluded that increased LARC uptake would reduce unintended pregnancy and be cost-effective. ${ }^{10}$ Only $12 \%$ of UK women aged 16-49 years used a LARC in 2008/09. ${ }^{9}$ Very long-acting contraception (vLARC) excludes injectable methods, which are the least cost-effective LARC. ${ }^{10}$ They require a woman to have an injection every 8-14 weeks and are therefore not as long-acting as implants and IUDs. ${ }^{10}$

An outcome of the national sexual health framework is "a reduction in the health inequalities gap in sexual health", with indicators including the rate of teenage pregnancy and abortion in areas of highest deprivation. ${ }^{5}$ There is still a significant gap in these indicators. While the effects of deprivation on abortion and teenage pregnancy rates are complex and multifactorial, it is important to determine if contraceptive prescribing is related to area deprivation, especially the most cost-effective methods (LARC).

Numerous studies have investigated contraceptive use and individual level socioeconomic factors. Women with lower education and social class by occupation are significantly less likely to use contraception in the UK, ${ }^{11}$ Europe ${ }^{12}{ }^{13}$ Australia ${ }^{14}$ and the USA. ${ }^{15}$ Wakhisi et al. ${ }^{16}$ analysed English Office for National Statistics data to explore factors associated with LARC use in women aged under 25 years. Before 2005 , women without qualifications were seven times more likely to use LARC compared with those with a degree. ${ }^{16}$ However, after LARC NICE guideline ${ }^{10}$ release there was no significant difference by education or occupation. ${ }^{16}$

Fewer studies have explored community level socioeconomic factors and contraceptive use. Bentley et al. ${ }^{11}$ analysed data from a large UK sample and after adjusting for individual and geographic variations, women in the lowest quintile of area disadvantage were $25-30 \%$ less likely to use any contraception than the most advantaged quintiles. ${ }^{11}$ However, the data were from the $1990 \mathrm{~s}$, a relatively crude measure of area deprivation was chosen, and the focus was on any contraceptive use rather than a specific type. Wakhisi and Barret $^{16}$ found no association between LARC uptake and area deprivation. The Scottish Health Survey 2008-2011 found that LARC use was higher in women living in the most deprived areas: $20 \%$ compared with $13 \%$ of women in the least deprived areas, ${ }^{17}$ but these were crude survey results without adjustment for potential confounders (e.g. age).

Using recent prescribing data from primary care, sexual health clinics and a combined dataset this study aimed to examine the relationship between area deprivation measured by the Scottish Index of Multiple Deprivation (SIMD) and LARC prescription for women of reproductive age in Lothian, Scotland adjusting for potential confounding effects.

\section{METHODS}

Electronic prescribing data from Lothian health board was examined. Since the majority of women obtain prescribed contraception from primary care and/or sexual and reproductive health (SRH) clinics, ${ }^{9}$ data from both sources was included. The Prescribing Information System (PIS) is the data source for contraceptives prescribed and dispensed in primary care in Scotland. ${ }^{18}$ Patient-level data regarding date of birth (DOB) and postcode is available for all prescriptions which record a unique Community Health Index (CHI) number. The Scottish National Sexual Health IT System $(\mathrm{NaSH})$ is an electronic patient record for SRH clinics in Scotland ${ }^{19}$ and also provides information for research including prescribing. An anonymous unique $\mathrm{NaSH}$ number is the identifier for each patient. Lothian has a central SRH service (Chalmers) with 11 peripheral clinics in areas with the highest level of sexual ill health and deprivation. ${ }^{20}$ Including both PIS and $\mathrm{NaSH}$ data is important to capture as complete a picture as possible. Although the majority of contraceptive prescriptions are from primary care, ${ }^{9}$ it is known that women attending SRH clinics are more likely to be prescribed LARC. ${ }^{10}$ Some general practitioners (GPs) do not provide all LARC methods or may be less likely to recommend them. ${ }^{10}$

Information was obtained for the whole population in the two datasets over the latest available 2-year period from 1 April 2012 to 31 March 2014 from each attendance in which females aged 13-54 years were prescribed contraception. Those women aged over 54 years were excluded because loss of fertility could be assumed. ${ }^{21}$ If a woman received more than one prescription in this period, the episode where the longest-acting method was prescribed was considered, in the following order: IUD/IUS > implant $>$ injectable $>$ short-acting hormonal (COCP, patch, ring) $>$ diaphragm. This methodology was chosen because access 
to a prescription of LARC was of primary interest. Emergency contraception and spermicide were excluded. Condoms were excluded as these are not prescribed.

$\mathrm{NaSH}$ and PIS data were combined for the main analysis. Since both systems use a different unique identifier and have different variables, a combined dataset has limitations. Over the time period, some women will appear in both datasets, having obtained prescriptions from both a SRH clinic and a GP. These women were identified by DOB and postcode and considered to be a 'match'. The episode with the longest-acting method was retained. However, two women may share the same postcode and DOB so cannot be differentiated from each other. Where duplicates existed based on DOB and postcode, these were removed from the combined dataset as it was not possible to discern which one should be matched with the other. Conversely, if a woman has changed postcode over the time period she will be double-counted.

Since the quality and accuracy of this matching process has not been reported previously, we conducted sensitivity analysis to explore this (the results of such analysis are presented in the Results section).

The study was approved by the NHS Lothian Caldicott Guardian. All patient-level data were extracted from the central database and cleaned by professional NHS Lothian data analysts to increase accuracy. This involved removing patient-identifiable data to comply with the request of the Caldicott guardian, as well as ensuring the NHS Lothian SIMD quintile was consistent over the 2 years of data. $\mathrm{NaSH}$ and PIS data were joined in a 'combined' dataset. Shown in Figure 1, 94515 women were in this dataset; 4365 $(4.6 \%)$ were excluded because they lacked a postcode, were non-Lothian residents or duplicates. The remaining 90150 women were the subjects of this analysis; $79639(88.3 \%)$ of these women obtained their prescription from primary care (PIS) and 10511
(11.7\%) from a SRH clinic (NaSH). Note that $\mathrm{NaSH}$ and PIS datasets examined individually (presented in Table 1) contained higher numbers since duplicates did not occur due to unique identifier numbers, so there were fewer exclusions.

The primary outcome (the dependent variable) was prescription of LARC (intrauterine, implant or injectable) versus non-LARC method (oral contraceptives, patch, ring and diaphragm). vLARC prescription (intrauterine methods and implants only) was a secondary outcome since it has relevance to Scottish targets and national statistics publications. Scottish Government prescribing targets relate to vLARC and Information Services Division Scotland produce reports with a focus on vLARC. ${ }^{5}$

The primary explanatory (independent) variable of interest was area deprivation measured by SIMD quintile. SIMD 2012 is the Scottish Government's official measure of area deprivation. ${ }^{22}$ It divides Scotland into 6505 small datazones and ranks them from 1 (most deprived) to 6505 (least deprived) to identify pockets of deprivation. Quintiles split up the data into five groups, each containing $20 \%$ of the data: quintile 1 is the most deprived and quintile 5 the least deprived. SIMD is subject to quality assurance ${ }^{23}$ and has been independently reviewed. ${ }^{24}$

As LARC and vLARC were dichotomous outcomes, logistic regression models were used to examine the relationships between SIMD and LARC, and between SIMD and vLARC, adjusting for potential confounding effects $^{25}$ of age and location of prescription (primary care or SRH clinic), depending on data availability in the combined dataset. In the analysis, the age range 13-54 years as a whole was examined. Narrower reproductive age ranges (i.e. 16-19, 20-29, 30-39 and $40-49$ years) were also examined. The age range subgroups were based in keeping with other publications, ${ }^{13} 1426$ not based on the distributions (sizes) of these subgroups of the data. Modelling both the

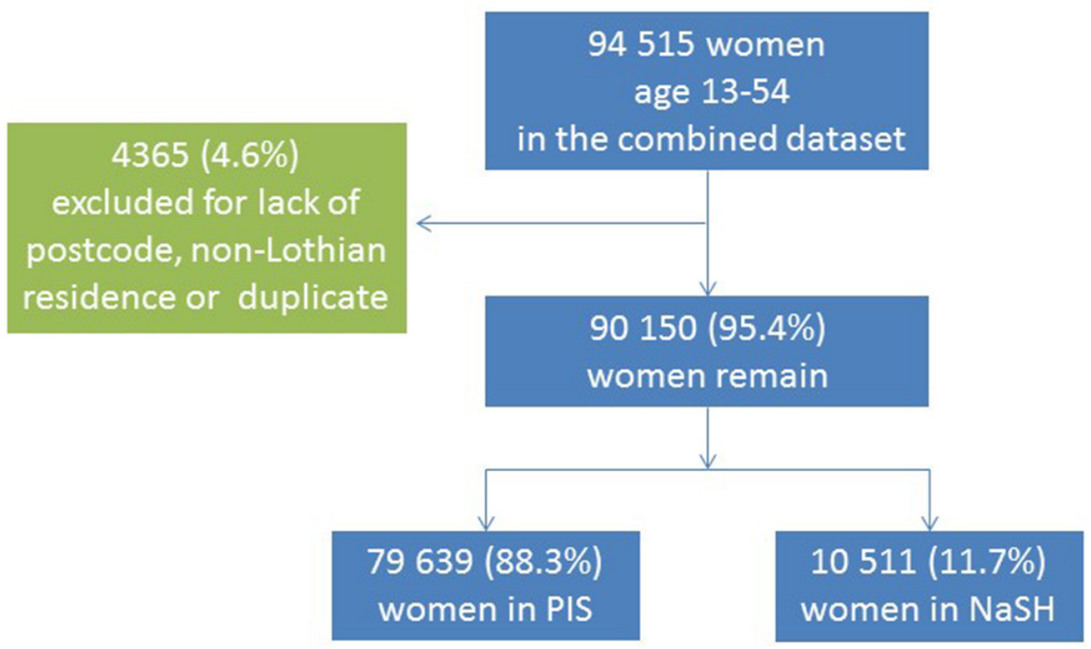

Figure 1 Flow diagram for women with prescribed contraception. NaSH, Scottish National Sexual Health IT System; PIS, Prescribing Information System. 
Table 1 Descriptive statistics

\begin{tabular}{|c|c|c|c|c|c|}
\hline Variable & & $\begin{array}{l}\text { Combined } \\
(n=90150)\end{array}$ & $\begin{array}{l}\text { NaSH } \\
(n=11190)\end{array}$ & $\begin{array}{l}\text { PIS } \\
(n=82263)\end{array}$ & $\begin{array}{l}\text { Comparison of } \\
\text { NaSH and PIS }\end{array}$ \\
\hline \multirow{2}{*}{$\begin{array}{l}\text { LARC } \\
\text { prescribed }\end{array}$} & Yes & $19033(21.1 \%)$ & $7559(67.6 \%)$ & $11888(14.5 \%)$ & $p<0.001$ \\
\hline & No & $71117(78.9 \%)$ & $3631(32.4 \%)$ & 70375 (85.5\%) & - \\
\hline \multirow[t]{2}{*}{ VLARC prescribed } & Yes & $13824(15.3 \%)$ & $7010(62.6 \%)$ & $\begin{array}{l}7049 \\
(8.6 \%)\end{array}$ & $p<0.001$ \\
\hline & No & $76326(84.7 \%)$ & $4180(37.4 \%)$ & $75214(91.4 \%)$ & - \\
\hline \multirow[t]{5}{*}{ SIMD quintile } & 1 (most deprived) & $18742(20.8 \%)$ & $2742(24.5 \%)$ & $16702(20.3 \%)$ & $p<0.001$ \\
\hline & 2 & $18997(21.0 \%)$ & $2481(22.2 \%)$ & $17201(20.9 \%)$ & $p=0.002$ \\
\hline & 3 & $18367(20.4 \%)$ & $2160(19.3 \%)$ & $16843(20.5 \%)$ & $p=0.004$ \\
\hline & 4 & $17099(19.0 \%)$ & $1914(17.1 \%)$ & $15698(19.1 \%)$ & $p<0.001$ \\
\hline & 5 (least deprived) & $16945(18.8 \%)$ & $1893(16.6 \%)$ & $15819(19.2 \%)$ & $p<0.001$ \\
\hline \multirow[t]{3}{*}{ Age (years) } & Range & $13-54$ & $13-54$ & $13-54$ & - \\
\hline & Mean & 28.6 (SD 8.9) & 29.3 (SD 9.4) & 28.4 (SD 8.8) & $p<0.001$ \\
\hline & Median & 27.0 & 27.0 & 27.0 & - \\
\hline \multirow[t]{6}{*}{ Age group (years) } & $<16$ & $2237(2.5 \%)$ & $296(2.7 \%)$ & $2010(2.4 \%)$ & $p=0.200$ \\
\hline & $16-19$ & $11733(13.0 \%)$ & $1370(12.2 \%)$ & $11094(13.5 \%)$ & $p<0.001$ \\
\hline & $20-29$ & $39787(44.1 \%)$ & $4753(42.5 \%)$ & 36493 (44.4\%) & $p<0.001$ \\
\hline & $30-39$ & $23298(25.8 \%)$ & $2754(24.6 \%)$ & $21235(25.8 \%)$ & $p=0.006$ \\
\hline & $40-49$ & $11818(13.1 \%)$ & $1807(16.1 \%)$ & $10329(12.6 \%)$ & $p<0.001$ \\
\hline & $50-54$ & $1277(1.4 \%)$ & $210(1.9 \%)$ & $1102(1.3 \%)$ & $p<0.001$ \\
\hline
\end{tabular}

LARC, long-acting reversible contraception; NaSH, Scottish National Sexual Health IT System; PIS, Prescribing Information System; SD, standard deviation; SIMD, Scottish Index of Multiple Deprivation; vLARC, very long-acting reversible contraception.

continuous variable and the subgroups of it is helpful to look for a trend as a whole while examining the behaviour of the specific subgroups at the same time.

Analyses were conducted using SPSS Version 16.0 (SPSS Inc., Chicago, IL, USA).

\section{RESULTS}

Table 1 presents descriptive information about the data.

Sensitivity analysis was conducted by performing exploratory analyses for each of the datasets (Combined, $\mathrm{NaSH}$ and PIS) separately as an approach to assessing the quality of the combined dataset. The 'Combined' dataset of 90150 women were the focus of this analysis. After exclusion for missing postcode or non-Lothian health board, the ' $\mathrm{NaSH}$ ' dataset contained 11190 women, while the 'PIS' dataset comprised 82263 women. We conducted hypothesis tests to compare PIS and NaSH for each pair of the corresponding groups in Table 1. For the continuous 'Age' variable, the hypothesis test was a comparison of the two means; for the remaining categorical variables, the hypothesis test was for a difference between the two proportions (percentages). The results ( $p$ values of the hypothesis tests) are presented in Table 1.

There was a clear difference in prescribing according to location of prescription, with SRH clinics having a significantly higher proportion of LARC/vLARC than primary care. This appears to support the logistic models adjusting for location of prescription. Overall, $21.1 \%$ of prescriptions were for LARC and $15.3 \%$ for vLARC. However, in primary care, LARC and vLARC proportions were lower at $14.5 \%$ and $8.6 \%$, respectively, and in SRH clinics there were much higher proportions of LARC (67.6\%) and vLARC (62.6\%). $\mathrm{NaSH}$ had a significantly higher proportion of women in the 40-49 years age group and more deprived women in Q1 and Q2. Conversely, PIS had significantly more women aged 16-39 years and in Q3-5.

Since NHS Lothian population weighted quintiles were used, it would be expected to find $20 \%$ of the study group in each deprivation (SIMD) quintile. However, on $z$-tests, there were significantly more in quintiles 1 [0.207 (95\% confidence interval (CI) 0.205-0.210)], 2 [0.211 (95\% CI 0.208-0.213)] and 3 [0.204 (95\% CI 0.201-0.206)] and fewer in quintiles 4 [0.190 (95\% CI 0.187-0.192)] and 5 [0.188 (95\% CI $0.185-0.191)]$.

Women aged 20-29 years accounted for the largest proportion of contraceptive prescriptions (44.1\%), which corresponds with other UK data, mainly because COCP use was proportionally higher in this group. ${ }^{9}$

Table 2 presents the results of the logistic regression models for LARC prescription on the 'Combined' dataset. A simple logistic model was run, regressing LARC prescription on deprivation (SIMD) only (results shown under the 'Unadjusted' column). We then ran a multiple logistic regression model of LARC 
Table 2 Model of long-acting reversible contraception prescription

\begin{tabular}{llll}
\hline Variable & & Unadjusted $0 R[(95 \% \mathrm{Cl}), \boldsymbol{p}$ value $]$ & Adjusted OR $[(95 \% \mathrm{Cl}), \boldsymbol{p}$ value $]$ \\
\hline SIMD quintile & 1 (most deprived) & 1 & 1 \\
& 2 & $0.84(0.80-0.88), p<0.001$ & $0.85(0.81$ to -0.90$), p=0.005$ \\
& 3 & $0.74(0.71-0.78), p<0.001$ & $0.76(0.72-0.81), p=0.001$ \\
& 4 & $0.59(0.56-0.63), p<0.001$ & $0.59(0.56-0.62), p<0.001$ \\
& 5 (least deprived) & $0.52(0.49-0.55), p<0.001$ & $0.51(0.48-0.55), p<0.001$ \\
Age & & - & $1.00(0.99-1.01), p=0.240$ \\
Age group (years) & $16-19$ & - & $1.02(0.95-1.00), p=0.910$ \\
& $20-29$ & - & 1 \\
Location & $30-39$ & - & $1.25(1.16-1.36), p<0.001$ \\
& $40-49$ & - & $1.59(1.38-1.83), p<0.001$ \\
\hline
\end{tabular}

$\mathrm{Cl}$, confidence interval; LARC, long-acting reversible contraceptive; NaSH, Scottish National Sexual Health IT System; OR, odds ratio; PIS, Prescribing Information System; SIMD, Scottish Index of Multiple Deprivation.

prescription and deprivation, adjusting for age, age group (restricted to reproductive age range 16-49 years) and location of prescription (results shown under the 'Adjusted' column).

The results show that increasing area deprivation is associated with increasing LARC prescription. Compared with Q1, Q2 is $15 \%$ less likely, Q3 is $24 \%$ less likely, Q4 is 41\% less likely and Q5 is 49\% less likely to receive a LARC prescription after adjusting for available potential confounders of age group and prescription location.

When women are grouped into age ranges it becomes apparent that those under 30 years are significantly less likely to be prescribed LARC. From age 30-49 years there is a significant increase in the likelihood of LARC prescription. Prescriptions from SRH clinics are over 13 times more likely to be LARC compared with those from primary care.
Table 3 presents the results of the logistic regression models for vLARC prescription on the 'Combined' dataset.

The results show similar messages. Compared with Q1, Q2 is 9\% less likely, Q3 is 10\% less likely, Q4 is $26 \%$ less likely and Q5 is 28\% less likely to receive a vLARC prescription after adjusting for age group and prescription location. Women aged 30-49 years are significantly more likely to be prescribed vLARC. Prescriptions from SRH clinics were over 19 times as likely to be for vLARC than those from primary care.

\section{DISCUSSION}

This analysis was based on a large dataset which includes almost all prescriptions issued in Lothian from primary care and SRH clinics: the two main sources of contraceptive prescriptions in the UK. ${ }^{9}$ Less than 5\% of cases were excluded. Prescriptions from secondary

\begin{tabular}{llll}
\hline \multicolumn{2}{l}{ Table 3} & Model of very long-acting reversible contraception prescription & \\
\hline Variable & & Unadjusted OR [(95\% Cl), $\boldsymbol{p}$ value] & Adjusted OR [(95\% Cl), $\boldsymbol{p}$ value] \\
\hline SIMD quintile & 1 (most deprived) & 1 & 1 \\
& 2 & $0.88(0.84-0.93), p<0.001$ & $0.91(0.86-0.97), p=0.005$ \\
& 3 & $0.83(0.79-0.88), p<0.001$ & $0.90(0.84-0.96), p=0.001$ \\
& 4 & $0.71(0.67-0.75), p<0.001$ & $0.74(0.69-0.79), p<0.001$ \\
Age & 5 (least deprived) & $0.67(0.63-0.71), p<0.001$ & $0.72(0.67-0.77), p<0.001$ \\
Age group (years) & $16-19$ & - & $1.02(1.01-1.02), p<0.001$ \\
& $20-29$ & - & $1.00(0.91-1.09), p=0.910$ \\
& $30-39$ & - & 1 \\
Location & $40-49$ & - & $1.30(1.18-1.42), p<0.001$ \\
& $\mathrm{PIS}$ & - & $1.53(1.29-1.80), p<0.001$ \\
\hline
\end{tabular}

$\mathrm{Cl}$, confidence interval; NaSH, Scottish National Sexual Health IT System; OR, odds ratio; PIS, Prescribing Information System; SIMD, Scottish Index of Multiple Deprivation; VLARC, very long-acting reversible contraceptive. 
care and private clinics are not included but account for a minority of prescriptions. ${ }^{9}$ Data were extracted from electronic recorded prescriptions, so recall bias was at least limited if not completely eliminated. Sensitivity analyses performed on individual $\mathrm{NaSH}$ and PIS datasets showed similar trends and drew the same conclusions that increasing deprivation was significantly associated with increasing LARC and vLARC. The data were recent, from 1 April 2012 to 31 March 2014, so should reflect current prescribing practice. The large majority (94\%) of women obtaining a contraceptive prescription over the 2-year period had only a single method type prescribed and this was similar between datasets. This implies that this time period was appropriate to analyse as a cross-sectional study.

The analysis confirms that there is a significant association between SIMD quintile and LARC and vLARC prescription. Of women prescribed contraception, increasing area deprivation is associated with increasing proportion of LARC and vLARC prescription, having adjusted for available potential confounders of age group and prescription location. These results are consistent with the Scottish Health Survey, which found that LARC use increased as the level of area deprivation increased: $13 \%$ of sexually active $16-55$-year-olds used a LARC in the least deprived quintile compared with $20 \%$ in the most deprived quintile from 2008-2011. ${ }^{17}$ Lothian was the target population. It should be noted that Lothian has fewer areas of deprivation compared with the West of Scotland. Greater Glasgow and Clyde health board has $45 \%$ of the national share of the $15 \%$ most deprived datazones in Scotland ${ }^{27}$ compared with Lothian's 7.5\% share. It would be interesting to examine and compare Lothian results to a more deprived health board such as Glasgow to see if the conclusions remain the same.

Age is a significant predictor of LARC prescription. Using the 20-29 years age group (the largest group) as a comparator shows a significantly increased likelihood of LARC prescription from age 30-49 years, reaching a peak at age $40-49$ years when there are 1.59 times odds of LARC, indicating that women aged 40-49 years are 59\% more likely to be prescribed LARC. For vLARC the pattern is similar. This is an expected result as women aged over 30 years are known to have higher use of LARC, particularly intrauterine methods. ${ }^{28} 29$ Women aged 20-34 years account for the majority of unplanned pregancies ${ }^{1}$ and the $20-24$ years age group has the highest abortion rate. ${ }^{8}$ There has been a focus on reducing teenage pregnancies, but less emphasis on the 19-24 years age group, who have been relatively neglected by teenage pregnancy strategies. ${ }^{7}$ Further research on how to increase uptake of LARC in this age range is needed.

Women prescribed contraception in SRH clinics are 13.5 times more likely to be prescribed a LARC and 19.4 times more likely to receive a vLARC method than those prescribed contraception in primary care.
This was expected since SRH clinics are known to prescribe a higher proportion of LARC methods. ${ }^{30}$ It will be interesting to see if primary care rates will increase now the cap on reimbursing GPs to fit vLARC in Lothian has been removed. ${ }^{31}$ In 2009 the Quality and Outcome Framework (QOF) incentivised GP contraceptive counselling for LARC. ${ }^{32}$ This payment for performance increased LARC prescribing rates by $4 \%$ annually. ${ }^{32}$ QOF has now been decommissioned in Scotland and previous research has indicated that when payment for performance is removed, performance can dip $^{33}$ or plateau. ${ }^{34}$

Patient-level data on SIMD quintile and age group was available for over 95\% of prescriptions. Variables (age and location of prescription) with potentially confounding effects on the relationships of deprivation and prescription of LARC and vLARC were available and hence could be controlled for in the logistic regression analyses, though it should be noted that other factors which are likely to be important such as parity and previous abortions, individual-level socioeconomic factors, distance and access to services, prescriber factors and social capital were not measured ${ }^{11}$ and could not be included in the analysis.

Prescription is assumed to equate to use by women which is not necessarily the case, and data are not included to show how long the method is used for. It was assumed that all prescriptions were used for contraception which may not be the case and thus overestimates contraception use, perhaps to a different degree between methods. In analysis, the longest-acting method a woman was prescribed during the study period was examined, which introduces bias as it may skew results towards LARC/ vLARC prescriptions. However, only $5.6 \%$ of the combined dataset were prescribed a second method so it seems unlikely this should have a large effect on the results.

Women included in this analysis have already accessed a prescription which could introduce bias since those who are less likely to access healthcare could be the most excluded and deprived groups. Women who are not using any contraception or those using condoms are not represented in the study. Of women ' at risk' of pregnancy, an estimated 5\% use no contraception and $37 \%$ use condoms. ${ }^{9}$ Women using no contraception are at high risk of pregnancy (85\% of women would be pregnant within 1 year), and with typical use of the male condom $15 \%$ would be pregnant in 1 year. ${ }^{10}$ These two groups may therefore account for a large proportion of unintended pregnancies. This is an important limitation of using a cross-sectional dataset of prescriptions. A UK survey study like the National Survey of Sexual Attitudes and Lifestyles (Natsal) would not have this limitation; ${ }^{35}$ however, data from the most recent survey (Natsal-3, 2010-2012) was not available. 


\section{CONCLUSIONS}

Using prescribing data from Lothian primary care, sexual health clinics and a combined dataset, results consistently show that women prescribed contraception residing in the most deprived quintile (Q1) have higher prescription rates of LARC and vLARC methods compared with those in less deprived quintiles. This could be due to higher patient demand, clinician prescribing behaviour or targeted peripheral SRH services, but indicates that women in more deprived quintiles who are prescribed contraception are accessing the most effective methods at least as well as less deprived areas. Since we do not have data on women with no prescription who are probably at higher risk of unplanned pregnancy, our results cannot inform whether higher rates of abortion and teenage pregnancy in areas of highest deprivation in Lothian are related to prescription of LARC methods compared with non-LARC methods in these areas.

There are implications for women in less deprived quintiles who are still at risk of unintended pregnancy and its potential consequences. Although 28\% of the 11475 abortions performed in Scotland in 2014 were for women residing in SIMD Q1, 23\% were for women in Q2, 19\% Q3, 16\% Q4\% and 14\% Q5. There is a spectrum of deprivation and the disadvantage of targeting the most deprived areas will be potential consequences to non-targeted areas. Further qualitative research is required to uncover the reasons behind these findings in more detail.

Although sexual health services are important, achieving higher proportions of LARC in women prescribed contraception in deprived areas does not necessarily result in reduced unintended pregnancies. Wider determinants of social inequalities such as education and employment also need to be targeted by policy to realise a narrowing of the inequality gap. ${ }^{36}$

Acknowledgements The authors would like to thank NHS Lothian Data Analysts Ms Eilidh Fletcher, Ms Ana Rodriguez and $\mathrm{Mr}$ Andrew Massie for their help in extracting and matching data. They would like to thank Ms Dona Milne, Deputy Director of Public Health and Health Policy at NHS Lothian, for agreeing to the project and the Caldicott Guardian Professor Alison McCallum, Director of Public Health and Health Policy at NHS Lothian for approval of the Caldicott application. The authors accept full responsibility for the analysis and interpretation of the data presented. This work formed part of CM's thesis towards the Masters in Public Health, University of Manchester.

Competing interests None declared.

Patient consent The author did not have access to any patient identifiable data. All the patient level data was analysed in a safe NHS Lothian Server. Aggregated data only left NHS Lothian once it had been aggregated and it complied with NHS Lothian Statistical Disclosure Guidance.

Ethics approval Caldicott guardian NHS Lothian.

Provenance and peer review Not commissioned; externally peer reviewed.

(C) Faculty of Sexual and Reproductive Healthcare of the Royal College of Obstetricians and Gynaecologists (unless otherwise stated in the text of the article) 2017. All rights reserved. No commercial use is permitted unless otherwise expressly granted.

\section{REFERENCES}

1 Wellings K, Jones KG, Mercer CH, et al. The prevalence of unplanned pregnancy and associated factors in Britain: findings from the third national survey of sexual attitudes and lifestyles (Natsal-3). Lancet 2013;382:1807-16.

2 Tsui AO, McDonald-Mosley R, Burke AE. Family planning and the burden of unintended pregnancies. Epidemiol Rev 2010;32:152-174.

3 Thomas CM, Cameron S. Can we reduce costs and prevent more unintended pregnancies? A cost of illness and costeffectiveness study comparing two methods of EHC. BMJ Open 2013;3:e003815.

4 Lucas S, (Brook and Family Planning Association). Unprotected Nation: The Financial and Economic Impacts of Restricted Contraceptive and Sexual Health Services: Development Economics Limited, 2013. http://www.fpa.org.uk/sites/default/ files/unprotected-nation-sexual-health-full-report.pdf. (accessed 15 July 2015).

5 The Scottish Government. The Sexual Health and Blood Borne Virus Famework 2011-15. Edinburgh, UK: Scottish Government, 2011. DPPAS11868. http://www.gov.scot/ Resource/Doc/356286/0120395.pdf. (accessed 31 June 2015).

6 Information Services Division Scotland. Teenage Pregnancy Year of Conception Ending 31st December 2013. National Statistics Publication for Scotland, 2015. http://www.isdscotland.org/ Health-Topics/Sexual-Health/Publications/2015-07-07/201507-07-TeenPreg-Report.pdf. (accessed 8 July 2015).

7 National Institute for Health and Care Excellence. Contraceptive Services With a Focus on Young People up to the Age of 25. NICE Public Health Guidance, 2014.

8 Information Services Division Scotland. Termination of Pregnancy Statistics Year Ending 31st December 2014. National Statistics Publication for Scotland, 2015. https://isdscotland. scot.nhs.uk/Health-Topics/Sexual-Health/Publications/2015-0526/2015-05-26-Terminations-2014-Report.pdf. (accessed 30 May 2015).

9 Lader D, (Office for National Statistics). Opinions Survey Report No. 41 Contraception and Sexual Heath, 2008/09 (41). NHS Information Centre for Health and Social Care, 2008.

10 National Institute for Health and Care Excellence (NICE). Long-acting Reversible Contraception: The Effective and Appropriate Use of Long-acting Reversible Contraception (NICE Clinical Guideline Volume 30).London, UK: NICE,2014.

11 Bentley R, Kavanagh A, Smith A. Area disadvantage, socioeconomic position and women's contraception use: a multilevel study in the UK. J Fam Plann Reprod Health Care 2009;35:221-6.

12 Layte R, McGee H, Rundle K, et al. Does ambivalence about becoming pregnant explain social class differentials in use of contraception? Eur J Public Health 2007;17:477-482.

13 Ruiz-Muñoz D, Pérez G, Garcia-Subirats I, et al. Social and economic inequalities in the use of contraception among women in Spain. J Womens Health 2011;20:403-411.

14 Ong J, Temple-Smith M, Wong WC, et al. Contraception matters: indicators of poor usage of contraception in sexually active women attending family planning clinics in Victoria, Australia. BMC Public Health 2012;12:1108.

15 Wu J, Meldrum S, Dozier A, et al. Contraceptive nonuse among US women at risk for unplanned pregnancy. Contraception 2008;78:284-289. 
Ending March 2014. National Statistics Publication Scotland, 2014. http://www.isdscotland.org/Health-Topics/Sexual-Health/ Publications/2014-09-30/2014-09-30-LARC-Report.pdf. (accessed 25 July 2015).

27 The Scottish Government. Scottish Index of Multiple Deprivation 2012. A National Statistics Publication Scotland, 2012. http://simd.scotland.gov.uk/publication-2012/localauthority-summaries-simd-2012/. (accessed 2 July 2015).

28 Cea-Soriano L, García Rodríguez LA, Machlitt A, et al. Use of prescription contraceptive methods in the UK general population: a primary care study. Bjog 2014;121:53-61.

29 Haimovich S. Profile of long-acting reversible contraception users in Europe. Eur J Contracept Reprod Health Care 2009;14:187-195.

30 Mansour D, Rosales C, Cox M. Women's awareness of long-acting, reversible contraceptive methods (LARCs) in community family planning clinics and general practice. Eur J Contracept Reprod Health Care 2008;13:396-399.

31 Hunter M. Long Acting Reversible Contraception (vLARC) Enhanced Service 2014/15. NHS Lothian: Primary Care Contractor Organisation.

32 Arrowsmith ME, Majeed A, Lee JT, et al. Impact of pay for performance on prescribing of long-acting reversible contraception in primary care: an interrupted time series study. PLoS One 2014;9:e92205.

33 Lester H, Schmittdiel J, Selby J, et al. The impact of removing financial incentives from clinical quality indicators: longitudinal analysis of four aiser ermanente indicators. BMJ 2010;340:c1898.

34 Kontopantelis E, Springate D, Reeves D, et al. Withdrawing performance indicators: retrospective analysis of general practice performance under UK ality and utcomes ramework. BMJ 2014;348:g330.

35 Erens B, Phelps A, Clifton S, et al. National Survey of Sexual Attitudes and Lifestyles 3. Technical Report Volume 1: Methodology. P2930, 2013. http://natsal.ac.uk/media/2090/ natsal-3-technical-report.pdf (accessed 1 August 2015).

36 Gomez AM, Fuentes L, Allina A. Women or LARC first? Reproductive autonomy and the promotion of long-acting reversible contraceptive methods. Perspect Sex Reprod Health 2014;46:171-175.

26 Information Services Division Scotland. Long Acting Reversible Methods of Contraception (LARC) - Key Clinical Indicator Year 